\section{GRP-054 DRUG SAFETY PROFILE: ANALGESICS AND ANAESTHETICS USED IN PAEDIATRIC ORTHOPAEDICS}

doi:10.1136/ejhpharm-2013-000276.054

M Falvo, L Trombetta, M Luppi, V Sassoli. Istituto Ortopedico Rizzoli, Farmacia Ospedaliera, Bologna, Italy

Background Pharmacological treatment of paediatric patients is a clinical area not deeply investigated because of the health, legal and financial implications. The multidisciplinary team for clinical management of drugs in Rizzoli Orthopedic Institute wanted to meet the clinical demand for standardisation of off-label use.

Purpose To develop a list of safe medicines for use in paediatrics. Materials and Methods The analysis was conducted considering the data sheets of 169 analgesics and anaesthetic medicines extracted from ATC M-N (60 drugs) and the following sources: TripDataBase, GUF for children 2003, BNF for Children 2011-2012, Who Model Formulary for Children 2010. The information stored in a DB enabled us to classify the medicines as: authorised in paediatrics; licenced with restrictions on use by age/weight/pathology; contraindicated or not recommended in childhood; with no references for use in children.

Results 30 anti-inflammatory/antirheumatic medicines (9 drugs) and 42 analgesics (10 drugs): ibuprofen, paracetamol and pethidine are reference drugs, ketorolac and nimesulide are contraindicated and there is no documentation for age $<16$ or $<12$ years; morphine has age limitations, but its use is strongly recommended; 12 muscle relaxants ( 9 drugs): suxamethonium, vecuronium, atracurium, baclofen, rocuronium are for reference; 31 anaesthetics (14 drugs): bupivacaine, isoflurane, remifentanil, ropivacaine and sevoflurane are authorised, thiopental and ketamine have no indications in childhood but their use is documented; 54 anticonvulsants (18 drugs), gabapentin and pregabalin are contraindicated for neuropathic pain although authorised over 6 years in epilepsy.

Conclusions The results confirmed the limited information contained in the data sheets and the need to apply scientific evidence in paediatrics. Therefore, the resulting list was a tool for clinicians to increase awareness of the off-label use as an aid in the acquisition of informed consent.

No conflict of interest.

\section{GRP-055 EFFECT OF ANTIVIRAL TREATMENT, VIRAL LOAD AND STAGE OF FIBROSIS IN OUALITY OF LIFE OF HEPATITIS C-INFECTED PATIENTS}

doi:10.1136/ejhpharm-2013-000276.055

P Nieto Guindo, H Mateo Carrasco, M Gimenez Ramos, J Fernandez Avila, FD Fernandez Gines. Hospital Torrecardenas, Pharmacy, Almeria, Spain

Background Some authors have reported reductions in healthrelated quality of life (HRQOL) of hepatitis C (HCV)-infected patients, but studies fail to discriminate between the effect of factors such as the antiviral regimen, the viral load (VL) or the degree of fibrosis.

Purpose To evaluate HRQOL in chronically-ill HCV patients prior to, and after, treatment initiation

Materials and Methods Inclusion criteria: patients >18-years old, HCV antibodies+ and HCV-RNA+, no other relevant comorbidities. Recruitment period: 9 months. Patients were stratified according to the previous VL and their degree of fibrosis, and started on antiviral treatment based on ribavirin + peginterferon. On their follow-up visits (weeks 0,4 and 12), subjects were given a validated questionnaire (SF36) to be completed at home and delivered on their next visit to the outpatient pharmacy. SPSS v17 was used for the statistical analysis.
Results 18 subjects recruited $(n=18)$, percentage of males $67 \%$, mean age 47.3. 10 patients had genotype 2 or 3 , and 8 patients had genotype 1 or 4. Low-grade (stage 1-2) and high-grade (3-4) fibrosis was found in 11 and 7 patients respectively. 9 patients had $>800,000$ RNA copies $/ \mathrm{mL}$ at presentation. With regard to the antiviral therapy, statistically significant differences in the following items were found between week 0 and week 4: physical functioning ( $\mathrm{P}=0.046)$, physical role $(\mathrm{P}=0.001)$, pain $(\mathrm{P}=0.001)$, health $(0.046)$, energy/ fatigue $(P=0.001)$, and emotional wellbeing $(P=0.001)$. Additionally, we found statistically significant differences in the emotional component with regard to the $\mathrm{VL}(\mathrm{P}=0.005)$ and the degree of fibrosis $(\mathrm{P}=0.03)$.

Conclusions Antiviral therapy was associated with deterioration in HRQOL. Items involving physical health exhibited the greatest differences. Conversely, those subjects with higher VL and an advanced degree of fibrosis had worse scores in the items involving emotional wellbeing. Long-term studies are currently being conducted to determine whether the existing differences are emphasised over time, as well as the implications of these findings.

No conflict of interest.

\section{GRP-056 EFFECT OF PHARMACEUTICAL FOLLOW-UP IN PATIENTS WITH SECONDARY HYPERPARATHYROIDISM TREATED WITH CINACALCET}

doi:10.1136/ejhpharm-2013-000276.056

FPA Fernández Pérez Ana, CPA Cahafeiro Pin Ana Isabel, LRI López Rodríguez Inmaculada, RLI Rodríguez Losada Isabel, LGV López García Víctor. Hospital Universitario Lucus Augusti, Farmacia, Lugo, Spain

Background Adherence in patients with hyperparathyroidism (sHPT) secondary to serious kidney disease treated with cinacalcet is very important for their health status.

Purpose To assess the adherence (percentage of days of treatment covered with medicine compared with the total days) observed in patients treated with cinacalcet and to evaluate the effect of the adherence reinforcement measures used with patients when medicine is dispensed in our Pharmacy Service (PS).

Materials and Methods Observational descriptive study (January 2012) of all patients treated with cinacalcet and selection of those with recorded lack of adherence. Tools: medical history, dispensing medicine record. To reinforce adherence the PS produced a brochure with recommendations, supplied pill boxes and designed a patient delay record to report to the medical doctor (MD). The results of adherence reinforcement were recorded in April, 2012.

Results From 66 patients treated with cinacalcet, we selected 13 $(19.7 \%)$ with a record of at least of 3 delays in collecting the prescription, 6 women and 7 men with a mean age of 65.3. Posology: $120 \mathrm{mg} / 24$ h $7.7 \%$ (1), 90 mg/24 h 15.4\% (2), 60 mg/24 h 23\% (3), $30 \mathrm{mg} / 24 \mathrm{~h} 38.5 \%$ (5), $30 \mathrm{mg} / 48 \mathrm{~h} 7.7 \%$ (1) and $30 \mathrm{mg} / 72 \mathrm{~h} 7.7 \%$ (1). The delay was between 4 and 70 days (15.5 on average). After adherence reinforcement measures, 6 patients collected their medicine punctually when it was next dispensed. After the second intervention, only 1 of the non-adherent patients came on time. All delays were communicated to the $\mathrm{MD}$.

Conclusions Although the doses are simple and the adherence support strategies applied are theoretically adequate, the results aren't satisfactory. Patients need to be informed of the repercussions of bad adherence and follow-up is needed with a combined strategy between the PS and the MD.

No conflict of interest. 